\title{
COMPETÊNCIA MORAL EM ESTUDANTES DE MEDICINA
}

Felipe Colombelli Pacca ${ }^{1}$, Patrícia Maluf $\mathrm{Cury}^{1}$, Stephanie Lee Basile Barboza Caseiro ${ }^{2}$, Thomas Portes de Almeida ${ }^{1}$, Carolina Colombelli Pacca Mazaro ${ }^{1}$, Tamara Veiga Faria ${ }^{1}$, Raul Aragão Martins ${ }^{2}$, Patrícia Unger Raphael Bataglia ${ }^{2}$

${ }^{1}$ Faculdade de Medicina em São José do Rio Preto - Faceres, Núcleo de Apoio Educacional e Psicológico Faceres, Curso de Medicina São José do Rio Preto, SP. ${ }^{2}$ Universidade Estadual Paulista - UNESP, Programa de Pós-Graduação em Educação, Marília, SP. E-mail: felipepacca@gmail.com

\section{RESUMO}

A reflexão autônoma e crítica sobre processos de tomada de decisão é objeto de construção também na educação superior. Tal capacidade é essencial para uma atuação profissional ética e pode ser operacionalizada como "competência moral", referindo-se à capacidade de emitir juízos baseados em princípios, mesmo em situações adversas nas quais o sujeito não encontre respaldo às suas opiniões. Essa avaliação simula situações complexas conflituosas e dilemáticas, frequentes na atuação profissional, especialmente daqueles que lidam com vidas humanas e relações sociais. Tomaremos como foco a competência moral dos estudantes de medicina. Os procedimentos metodológicos foram pautados em três pontos: a formação ética do estudante, o papel do aluno no processo de formação pleno, e o professor enquanto elemento importante para o desenvolvimento da moral do estudante. Os resultados apontam para a necessidade de desenvolvimento de estudos sobre o tema e para a construção e acompanhamento da moral de estudantes de medicina.

Palavras-Chave: Competência Moral, Reflexão Crítica, Educação Superior, Educação Médica.

\section{MORAL COMPETENCE IN MEDICAL STUDENTS}

\begin{abstract}
Autonomous and critical reflection on decision-making processes is also the object of construction in higher education. Such capacity is essential for professional ethical action and can be operationalized as "moral competence", referring to the ability to make judgments based on principles, even in adverse situations in which the subject does not find support for their opinions. This evaluation simulates complex conflicting and dilemmatic situations, frequent in professional practice, especially those dealing with human lives and social relations. We will focus on the moral competence of medical students. The methodological procedures were based on three points: the ethical training of the student, the role of the student in the process of full training, and the teacher as an important element for the development of student morale. The results point to the need to develop studies on the subject and for the construction and monitoring of the morale of medical students.
\end{abstract}

Keywords: Moral Competence, Critical Reflection, Higher Education, Medical Education. 


\section{INTRODUÇÃO}

Os dilemas morais são problemas vividos que colocam em conflito ao menos dois princípios de igual ou semelhante importância, irreconciliáveis e que exigem uma decisão. Tais dilemas tendem a ser mais complexos de acordo com o desenvolvimento do ser. A sociedade é um conjunto de relações sociais e, portanto, não há como homogeneizar a moral prescrita a um indivíduo. É a partir da interação social que a moral é desenvolvida, visto que as situaçõesproblema em que se evidencia tal desenvolvimento, há sempre ao menos dois elementos. Porém, quando se discute o desenvolvimento da autonomia, a maioria das pesquisas versa sobre o desenvolvimento infantil ou infanto-juvenil, a partir do proposto por Piaget (Piaget, 1994; Kawashima et al., 2015).

No caso do adulto, espera-se que a autonomia já seja uma realidade no indivíduo e, portanto, também é esperado que exista consciência de suas decisões morais. Isso é, o adulto deve ser apto a analisar fundamentos ou razões para determinadas decisões. Para ser autônomo, ele deve ter desenvolvida sua capacidade de reflexão crítica e sua competência moral (Ferreira, 2016). Assim, para se tornar uma pessoa crítica sobre o próprio desenvolvimento moral, é preciso que as situações complexas vivenciadas (ou imaginadas) tornem-se fruto de reflexão pessoal. É preciso ir além do que se ouve, do que se vê, para que uma decisão seja tomada.

Por "desenvolvimento moral", nos referimos especificamente à perspectiva desenvolvimentista de Lawrence Kohlberg (1981, 1984). Kohlberg desenvolvera sua teoria com base em princípios construtivistas de Jean W. F. Piaget (1932/1994) e afirma que o desenvolvimento moral ocorre de forma análoga e co-dependente ao desenvolvimento cognitivo. Análoga no sentido de depender igualmente das trocas realizadas entre o indivíduo e o meio e co-dependente pois afirma que o desenvolvimento moral tem como condição necessária o desenvolvimento cognitivo, ainda que este não seja condição suficiente. (Ferreira, 2016)

Este processo de desenvolvimento moral não é finalizado durante a vida. Não existe uma linha de chegada para o desenvolvimento da moral, na qual uma pessoa torna-se premiada e vitoriosa nesse elemento. Na verdade, é um processo que, conforme as situações são vivenciadas, mais complexo se torna. Assim, desde a decisão sobre estudar ou não para uma prova de química durante a fase final do período escolar, passando pela escolha da profissão, relacionamentos conjugais, criação ou não de filhos, vivências durante a terceira idade, são todas possibilidades de situações que podem determinar maior ou menor complexidade para o desenvolvimento da moral do indivíduo no decorrer de sua vida. Um exemplo é o curso de medicina. Neste curso, os discentes são confrontados a dilemas morais bastante importantes, como, por exemplo, decisões que acarretem morbidades, sequelas ou até mesmo a morte de um paciente. Porém, a literatura aponta para um despreparo para tais vivências tanto do aluno e quanto das escolas de medicina (Rego, 2012; Feitosa et al., 2013; Landim et al., 2015).

A formação ética apresenta-se como elemento importante para a excelência do futuro da profissão (Rego e Schillinger-Agatti, 2011) e exigência de formação de médicos generalistas e humanistas (Brasil, 2005; 2014). Torna-se então relevante a investigação do processo de reflexão crítica dos alunos e na intervenção didática que direcione o aluno para uma prática moral e ética mais eficiente.

Nesse contexto, o objetivo deste estudo é discutir o desenvolvimento da competência moral do estudante de medicina evidenciando três aspectos: 1) A ética e a formação humana do estudante de medicina; 2 ) 0 papel do estudante enquanto protagonista da construção de sua autonomia; 3) A prática docente no curso de medicina e suas implicações para o desenvolvimento ético dos alunos. 


\section{METODOLOGIA}

Foram selecionados textos a partir de pesquisas bibliográficas e bases de dados do Banco Virtual de Saúde (BVS), EBSCO. Os três aspectos identificados como elementos de discussão para o estudo foram identificados em uma análise conceitual de pesquisa por levantamento, documental, transversal, na perspectiva do Desenvolvimento Moral proposto por Piaget (Piaget, 1994) e ampliado por Kohlberg (Kohlberg, 1984). O processo de análise do material seguiu, de acordo com (Cozby, 2012), primeiro identificando conceitos abrangentes e, em seguida, buscando compreender conceitos específicos.

\section{RESULTADOS}

Os resultados apresentados a seguir foram divididos em três diferentes elementos, sendo: 1) A ética e a formação humana do estudante de medicina; 2) 0 papel do estudante enquanto protagonista da construção de sua autonomia; 3) A prática docente no curso de medicina e suas implicações para o desenvolvimento ético dos alunos.

\section{DISCUSSÃO}

\section{A ÉTICA E A FORMAÇÃO HUMANA DO ESTUDANTE DE MEDICINA}

A formação ética dos profissionais de saúde, especificamente dos médicos, principalmente no Brasil, é superficial (Rego, 2012). Rego afirma

Não tenho a menor dúvida em afirmar que a maioria das faculdades de medicina, tal como são organizadas e buscam cumprir a função primordial que justifica a sua existência - preparar jovens para o exercício da profissão médica -, é incompetente. Da mesma forma, peremptória, afirmo que, em qualquer lugar do mundo, a incompetência da maioria das escolas é semelhante. Se no campo do desenvolvimento das habilidades técnicas ou da competência técnica existem várias escolas no mundo que têm uma atuação satisfatória, no desenvolvimento das competências, digamos relacionais, como as de comunicação e éticas, elas deixam muito a desejar. (Rego, 2012)

Assim, a formação do médico, mesmo em instituições que utilizam metodologias de ensino e aprendizagem mais contemporâneas, ainda é bastante distante do ideal. Rego diz que essa é uma questão importante porque a educação médica não pode "permanecer nas mãos de profissionais ignorantes em pedagogia, ainda que bons técnicos, muito menos ser relegada à educação moral" (Rego, 2012).

A formação humana do estudante de medicina tem sido foco de estudos nos últimos anos, com investigações sobre a moral do estudante de medicina (Feitosa et al., 2013), discussões sobre a formação de um "bom médico" (Gordon, 2017), que apontam que os médicos em formação percebem seu ambiente de formação pela comunicação e interações de linguagem e, a partir desses elementos, vão dando forma à sua identidade de profissionais da saúde.

Porém, Lucchetti aponta que

O médico, antes muito respeitado pela sociedade e pacientes, vem cada vez mais perdendo seu espaço na sociedade. Não é incomum ver médicos agredidos, médicos que fraudaram exames, atestaram pessoas que nunca viram, negligenciaram os pacientes ou atendem em dois lugares ao mesmo tempo. (...) Esse reflexo da medicina atual, como não poderia deixar de ser, tem um impacto profundo em nossos estudantes de medicina. (Lucchetti, 2016) 
Serodio aponta que a ética está relacionada com escolhas que o indivíduo faz (Serodio et al., 2016a), quando discute a relação de problemas práticos. Para ele, os problemas morais são reflexos dos problemas éticos e surgem de "conflitos entre visões diferentes do que constitui uma vida boa". A formação ética e humanizada do futuro médico, então, sofre um processo de discussão e tentativas importantes para garantir o futuro da medicina ética no Brasil. Partindo do princípio que o médico, enquanto profissional, assumirá um papel decisivo nos locais de saúde, o estudo de Espinha aponta para o despreparo do recém-formado em desenvolver plenamente suas funções (Espinha, 2016), pois discute as diferenças de processos pedagógico-críticos que a faculdade foca, em detrimento de outros aspectos ético-filosóficos que também deveria desenvolver. Espinha diz que

Não é o 'doutor' bordado num jaleco que dará todo respeito que se pode conquistar numa relação profissional, mas aquilo que se faz para ter o verdadeiro respeito e admiração num encontro de palavras e ações (Espinha, 2016)

A formação ética do futuro médico é parte integrante da composição de sua reflexão crítica. Serodio acredita que o aluno, ao ser apresentado aos fundamentos filosóficos da moral, de compreender os princípios da ética e ter contato com modelos para reflexão bioética, ele poderia desenvolver a sensibilidade e o raciocínio moral (Serodio e Almeida, 2009). É possível então, inferir que é a partir da reflexão crítica que o comportamento ético do estudante de medicina é forjado. Taille crê que é a por intermédio das opções éticas que a motivação moral se flexibiliza e dá conta das várias condutas que se observam (Taille, 2010).

Nesse contexto, é importante que as instituições de ensino médico estejam atentas ao trabalho pedagógico que desenvolvem. A ética, a moral e a reflexão crítica de todo o processo de formação devem ser foco e ter espaço (também) em currículos de cursos. Em estudo que discutiu a rede social dos alunos em uma faculdade de medicina, Isba, Woolf e Hanneman apontam que esse é um caminho para explorar trabalho em equipe e, dessa maneira, preparar os futuros médicos para uma realidade profissional mais próxima do concreto e mais distante da idealizada por eles (Isba et al., 2017). Nesse contexto, o mau comportamento dos alunos deixa de ser avaliado exclusivamente como algo que atrapalhe seu rendimento acadêmico, mas passa a ser avaliado de acordo com seu comportamento moral, o que, para Lind, não é fruto de intenções ruins, mas da incapacidade de transformar as boas intenções em boas ações (Lind, 2007).

\section{O PAPEL DO ESTUDANTE ENQUANTO PROTAGONISTA DA CONSTRUÇÃO DE SUA AUTONOMIA}

Parte do processo de formação do futuro médico é de responsabilidade da instituição que ele frequentar, parte das experiências que os professores podem proporcionar ao aluno, mas grande parte é resultado direto do que o próprio aluno faz de seu processo de formação. Sem refletir criticamente sobre sua formação, ou seja, sem dominar sua competência moral, o estudante fica à mercê de diversas situações que dificultam sua formação, tais como a falta de motivação para os estudos, burnout, depressão e desvalorização pessoal e da profissão (Paula et al., 2014; Perrella, 2017). Algumas atividades propostas para os estudantes fazem com que o processo de autonomia de formação aconteça mais tranquilamente. Para isso, sugere atividades focadas em empatia, perspectiva e resiliência (Perrella, 2017; Vliet et al., 2017). No entanto, se o aluno não tiver competência moral para avaliar a importância e consequências das atividades para seu processo de formação, questiona-se a necessidade de tais atividades.

O desenvolvimento da competência moral deve ser uma preocupação na formação do médico. Isso pode se dar pela relação com outros alunos e suas atitudes enquanto estudantes (Berkhout et al., 2017). Desenvolver a capacidade de reflexão crítica dos alunos para que possam compreender a importância e relevância do processo de formação para o enfrentamento da 
realidade profissional torna-se importante para questionar o modelo de ensino que está proposto em diversas escolas de medicina do Brasil (Liboni; Siqueira, 2009). Rego explica que a questãochave para a análise do grau de desenvolvimento moral de um sujeito é a capacidade dele próprio, individualmente, analisar racional e autonomamente os problemas morais, independentemente dos valores que ele assume como seus, e decidir. (Rego, 2012).

A formação da competência moral deveria ser fonte de preocupação das instituições. Serodio et al. já explicavam que os estudantes não deveriam aprender apenas teorias e práticas médicas, mas também sobre como se comportar como um médico (Serodio et al., 2016b). Rego também atenta para "o interesse tanto do entendimento do como se processa o desenvolvimento da capacidade de raciocinar moralmente como os valores que são transmitidos através da socialização profissional" (Rego, 2012). E esse comportamento de médico é fonte de reflexão crítica desenvolvida por meio de experiências durante a faculdade, que passa, inclusive, pela noção de justiça, de certo e errado, de escolhas e posicionamentos éticos, de vida e morte (Marchi e Hossne, 2012; Rego, 2012).

O futuro médico deve compreender, para que exista a possibilidade de identificação e aproveitamento de situações importantes para a sua competência moral, que é ele o protagonista de seu desenvolvimento moral e de sua capacidade de reflexão crítica. Puig apresenta, entre outras informações sobre a formação do perfil da personalidade moral, que nosso cérebro tenta resolver uma gama variada de problemas sócio-morais adotando critérios elaborados culturalmente e aplicando soluções condutuais prescritas socialmente (Puig, 1998). Ou seja, é próprio indivíduo o responsável pela sua capacidade moral reflexiva. A compreensão da competência moral deve ser protagonizada pelo próprio estudante. Este, munido dessa capacidade, poderá identificar, treinar e desenvolver, durante seu processo de formação, sua capacidade de reflexão crítica.

\section{A PRÁtICA DOCENTE NO CURSO DE MEDICINA E SUAS IMPLICAÇÕES PARA O DESENVOLVIMENTO ÉTICO DOS ALUNOS}

O professor também exerce papel importante na formação ética do estudante de medicina. Serodio explica que

Em meados da década de 1980, o Conselho Regional de Medicina do Estado de São Paulo (CREMESP) promoveu uma série de debates entre especialistas para levantar os problemas relacionados à formação moral na graduação médica. O principal problema apontado foi justamente o mau exemplo dado pelos médicos durante o atendimento. (Serodio, 2006)

Serodio defende que é a partir da concepção sobre a participação docente no processo de formação ético-moral dos estudantes de medicina, haja visto que a maioria dos professores entrevistados consideravam que o estudante não chegava à faculdade totalmente autônomo em relação ao seu caráter moral (Serodio; Almeida, 2009). Nesse sentido, direcionar esforços para a elaboração de currículos com mais foco no desenvolvimento da competência moral do que os currículos atuais é tão importante quanto a postura docente que o professor apresenta em sala e durante as práticas (Serodio, 2013; Serodio et al., 2016a; b).

Os valores tornam-se, então, fonte de ensino e aprendizagem na formação médica. É papel do professor identificar, avaliar e desenvolver a reflexão crítica de seus alunos, seja incentivando a discussão de valores como elementos de desenvolvimento profissional (Patterson et al., 2015), seja pela discussão sobre como elaborar melhor a proposta didático-científica utilizada para ensinar, de acordo com uma abordagem baseada na ciência da aprendizagem (Gooding et al., 2017), seja pelo cuidado com a importância do estabelecimento ético da relação médico-paciente no processo de formação do médico (Grosseman; Karnopp, 2011). 
Rego explica que a relação entre professor e aluno deveria ser pautada no respeito mútuo e na preocupação consciente do professor sobre a influência que terá na formação do aluno (Rego, 2012). Assim também é o papel do professor, mesmo que sejam poucos aqueles que realmente se envolvem no processo de formação de seus alunos para que, no futuro, exerçam adequadamente sua função (Rego, 2012).

Dessa forma, o processo reflexivo dos alunos deve ser pautado, entre outros elementos, no professor preparado para discutir criticamente a formação dos estudantes. Preparo este que difere prática e teoria, que transpõem didaticamente as experiências realmente importantes para a formação de um bom médico daquelas consideradas desagradáveis (Rego, 2012). A educação opera, inclusive, em produções do cotidiano e aposta em novas relações por meio de interações, buscando construir a aceitação do estudante à mudança proposta pelo professor, que se transformará em práticas e novos conhecimentos pelos alunos (Ceccim; Ferla, 2011), inclusive conhecimentos e práticas de relacionamento, de atitudes e de reflexão crítica.

\section{CONCLUSÃO}

Desde 2009, o Conselho Nacional de Saúde, a Associação Brasileira de Ensino Médico e o Conselho Federal de Medicina vêm buscando um novo direcionamento no processo de formação dos médicos brasileiros, focando os esforços para a modificação do sistema de ensino e aprendizagem baseados em metodologias ativas e na formação de um profissional generalista e humanista, em detrimento da formação especializada que era comum nos cursos até então. Em 2014, as novas Diretrizes Curriculares Nacionais do Curso de Graduação em Medicina (Brasil, 2014) em consonância com a Resolução no 350 do Conselho Nacional de Saúde (Brasil, 2005) concretizaram o direcionamento iniciado em 2009.

Direcionar atividades didáticas de formação médica, como forma de auxílio na estruturação dos novos currículos, serão muito importantes, principalmente nos cursos abertos em locais distantes e ermos, pois é na ciência que debruçar-se-ão os futuros professores e alunos para embasar suas práticas. A formação ética, nesse contexto, apresenta-se como elemento importante para a excelência do futuro da profissão (Rego; Schillinger-Agatti, 2011) e exigência de formação de médicos generalistas e humanistas (Brasil, 2005; 2014).

Nota-se, então, a relevância deste estudo na investigação do processo de reflexão crítica dos alunos e na intervenção didática que direcione o aluno para uma prática moral e ética mais eficiente. É a partir das intervenções éticas no processo de formação que o estudante confrontará sua própria consciência moral e atitudes. É nesse ambiente que o raciocínio moral específico da profissão médica terá oportunidade de ser exercitado pelos alunos. Enfim, são os momentos ricos de situações dilemáticas e complexas que proporcionam maior desenvolvimento da competência moral. Dessa maneira, cabe ao aluno, enquanto protagonista de seu desenvolvimento, aproveitar as oportunidades de exercício da reflexão crítica, e cabe ao professor, enquanto elemento essencial na promoção dessas atividades, proporcionar ao aluno as oportunidades de desenvolvimento, pois é a partir do desenvolvimento ético que a reflexão crítica é forjada.

\section{REFERÊNCIAS}

BERKHOUT, J. J. et al. How clinical medical students perceive others to influence their selfregulated learning. Medical Education, v. 51, n. 3, p. 269-279, 2017.

BRASIL. RESOLUÇÃO № 350, DE 09 DE JUNHO DE 2005. CONSELHO NACIONAL DE SAÚDE: MINISTÉRIO DA SAÚDE 2005. 
. RESOLUÇÃO № 3, DE 20 DE JUNHO DE 2014. Diretrizes Curriculares Nacionais do Curso de Graduação em Medicina. CONSELHO NACIONAL DE EDUCAÇÃO. CÂMARA DE EDUCAÇÃO SUPERIOR: MINISTÉRIO DA EDUCAÇÃO 2014.

CECCIM, R. B.; FERLA, A. A. Abertura de um eixo reflexivo para a educação da saúde: o ensino e o trabalho. In: MARINS, J. J. N. e REGO, S. (Ed.). Educação médica: Gestão, cuidado, avaliação. São Paulo: Hucitec, 2011. cap. 12, p.258-277. ISBN 978-85-7970-123-8.

COZBY, P. C. Métodos de pesquisa em ciências do comportamento. 1. São Paulo: Atlas, 2012. 454 ISBN 978-85-224-3363-6.

ESPINHA, D. C. M. O trabalho com outras profissões: Reflexões acerca do cuidado em equipe. In: SILVA, A. F. D.;JUNIOR, D. I., et al (Ed.). Uma nova medicina para um novo milênio: A humanização do Ensino Médico. São Paulo: AME Editora, 2016. p.93-112. ISBN 978-85-87052-07-0.

FEITOSA, H. N. et al. Competência de Juízo Moral dos Estudantes de Medicina: um Estudo Piloto. Revista Brasileira de Educação Médica, v. 37, n. 1, p. 5-14, 2013, https://doi.org/10.1590/S0100$\underline{5022013000100002 .}$

FERREIRA, L. A. K. A relação entre capacidade reflexiva, crenças, valores e ambiente formador: Um estudo sobre a competência moral de estudantes da pós-graduação em educação. 2016. 133 (Mestrado em Ciência da Educação). Programa de Pós-graduação em Educação, Universidade Estadual Paulista Julio de Mesquita Filho, Faculdade de Filosofia e Ciências - Campus Marília.

GOODING, H. C.; MANN, K.; ARMSTRONG, E. Twelve tips for applying the science of learning to health profession education. Medical Teacher, v. 39, n. 1, p. 26-31, 2017, https://doi.org/10.1080/0142159X.2016.1231913.

GORDON, L. J. Moving beyond being a 'good doctor' to thinking about 'good doctoring processes'. Medical Education, v. 51, n. 3, p. 237-238, 2017, https://doi.org/10.1111/medu.13245.

GROSSEMAN, S.; KARNOPP, Z. M. P. Relação médico-paciente, terapêutica e a formação médica: a ética, a cidadania e o cuidado nas interações. In: MARINS, J. J. N. e REGO, S. (Ed.). Educação médica: Gestão, cuidado, avaliação. São Paulo: Hucitec, 2011. cap. 8, p.131-173. ISBN 978-857970-123-8.

ISBA, R.; WOOLF, K.; HANNEMAN, R. Social network analysis in medical education. Medical Education, v. 51, n. 1, p. 81-88, 2017, https://doi.org/10.1111/medu.13152.

KAWASHIMA, R. A.; MARTINS, R. A.; BATAGLIA, P. U. R. Histórias e dilemas morais com crianças: Instrumento para pesquisadores e educadores. Interfaces da Educação, v. 6, n. 16, p. 211-230, 2015. ISSN 2177-7691.

KOHLBERG, L. The Psychology of Moral Development. San Francisco: Harper \& Row Publisher, 1984.

LANDIM, T. P. et al. Competência de Juízo Moral entre Estudantes de Odontologia. Revista Brasileira de Educação Médica, v. 39, n. 1, p. 41-49, 2015, https://doi.org/10.1590/1981$\underline{52712015 \mathrm{v} 39 \mathrm{n} 1 \mathrm{e} 01612014 .}$ 
LIBONI, M.; SIQUEIRA, J. E. Competência moral do estudante de medicina. Revista da Associação Médica Brasileira, v. 55, n. 2, p. 226-228, 2009, https://doi.org/10.1590/S0104-42302009000200031.

LIND, G. La moral puede enseñarse: manual teórico-práctico de la formación moral y democrática. México: Trillas, 2007.

LUCCHETTI, G. Ética do estudante de medicina: Uma reflexão. In: SILVA, A. F. D.;IUNIOR, D. I., et al (Ed.). Uma nova medicina para um novo milênio: A humanização do Ensino Médico. São Paulo: AME Editora, 2016. p.85-92. ISBN 978-85-87052-07-0.

MARCHI, N. M. G. C. D.; HOSSNE, W. S. Reflexões bioéticas: o que pensam estudantes de medicina sobre o início e final da vida. Revista Bioethikos - Centro Universitário São Camilo, v. 6, n. 3, p. 271286, 2012.

PATTERSON, F. et al. What are values and how can we asses them? Implications for values-based recruitment in healthcare. In: CLELAND, J. e DURNING, S. J. (Ed.). Researching Medical Education. 1. Oxford: Association for the Study of Medical Education, 2015. cap. 15, p.167-180. ISBN 978-1118-83920-1.

PAULA, J. D. A. D. et al. Prevalência e fatores associados à depressão em estudantes de medicina. Journal of Human Growth and Development, v. 24, n. 3, p. 274-281, 2014.

PERRELLA, A. Standing in quicksand: The triad of 'perspective-empathy-resiliency' in medical education. Medical Education, v. 51, n. 3, p. 241-243, 2017, https://doi.org/10.1111/medu.13215.

PIAGET, J. O juízo moral na criança. São Paulo: Summus, 1994.

PUIG, J. M. Perfil da Personalidade Moral. In: (Ed.). A construção da personalidade moral. São Paulo: Ática, v.140, 1998. p.77-147.

REGO, S. A formação ética dos médicos: saindo da adolescência com a vida (dos outros) nas mãos. Rio de Janeiro: Editora FIOCRUZ, 2012. 169 ISBN 85-7541-021-0.

REGO, S.; SCHILLINGER-AGATTI, M. Desenvolvimento moral e ambiente de ensino-aprendizagem nas escolas médicas. In: MARINS, J. J. N. e REGO, S. (Ed.). Educação médica: Gestão, cuidado, avaliação. São Paulo: Hucitec, 2011. cap. 7, p.114-130. ISBN 978-85-7970-123-8.

SERODIO, A. M. D. B. Concepções e práticas dos profissionais que exercem atividade docente sobre seu papel na formação ético-moral do estudante de medicina da Unifesp. 2006. 143 Dissertação (Mestre em Ciências da Saúde). Programa de Pós-Graduação em Ensino em Ciências da Saúde, Universidade Federal de São Paulo, Escola Paulista de Medicina.

. Avaliação da competência do juízo moral de estudantes de medicina: comparação entre um curso de bioética tradicional e um curso de bioética complementado com o método Konstanz de Discussão de Dilemas: a educação em bioética na promoção das competências moral e democrática de adultos jovens. 2013. 137 Tese (Doutor em Ciências da Saúde). Programa de PósGraduação em Pediatria, Universidade Federal de São Paulo, Escola Paulista de Medicina. 
SERODIO, A. M. D. B.; ALMEIDA, J. A. M. D. Do humanismo à ética: concepções e práticas docentes na promoção do desenvolvimento moral do estudante de medicina. Revista Bioética, v. 17, n. 2, p. 281-296, 2009.

SERODIO, A. M. D. B.; KOPELMAN, B. I.; BATAGLIA, P. U. R. Promoting moral and democratic competencies: towards an educational turn of Bioethics. Revista Bioética, v. 24, n. 2, p. 235-242, 2016a, https://doi.org/10.1590/1983-80422016242123.

- The promotion of medical students' moral development: a comparison between a traditional course on bioethics and a course complemented with the Konstanz method of dilemma discussion. International Journal of Ethics Education, v. 1, p. 81-89, 2016b, https://doi.org/10.1007/s40889-016-0009-8.

TAILLE, Y. D. L. Moral e Ética: Uma leitura psicológica. Psicologia: Teoria e Pesquisa, v. 26, n. n. Especial, p. 105-114, 20102010.

VLIET, M. V.; JONG, M.; JONG, M. C. Long-term benefits by a mind-body medicine skills course on perceived stress and empathy among medical and nursing students. Medical Teacher, 2017. ISSN 0142-159X. 